\title{
From System Thinking to Capability Thinking using the Thinking Capability Analysis Technique
}

\author{
Hussein A Abbass ${ }^{a}$ and Leon Young

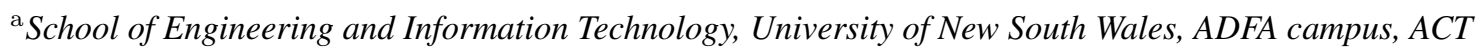 \\ 2600, Australia \\ Email: $\underline{\text { h.abbass@adfa.edu.au }}$
}

\begin{abstract}
System Thinking (ST) attempts to understand how different system components come together to form the overall whole of a system. This has proven to be a very valuable approach to study and understand complex systems; especially, complex socio-technical systems. In this context, a system exists for a purpose. This purpose is usually defined in the eyes of the beholders: external observers that need to make a judgement on what the purpose of the system is.

A capability is the capacity to generate an effect. It is made up of building-blocks of systems known as fundamental inputs to capabilities (FICs). In essence, a capability is a system of systems. A capability definition departs from a system definition in that the purpose of a capability (effect generation) is an integral part of the definition; that is, a capability can't be defined in isolation of the effects it is meant to generate. In essence, the type of effects a capability needs to generate needs to be part of the design and should not be merely left for an external observer's judgement. Therefore, CApability Thinking (CAT) focuses on how systems build the energy and capacity required in a system of systems to generate effects.

In this paper, CAT will be explored and discussed through similarities and differences to ST. We will then present The Thinking Capability Analysis Technique (TCAT) which extends the Function Analysis System Technique (FAST) in System Engineering. FAST has demonstrated a great deal of success as a decision aiding tool to map out functional interdependency for Requirement Engineering. TCAT replaces the how question in FAST with enablers, it extends the notations to include responsibility or the whom question to simultaneously establish a line of accountability, and increases the flexibility of the representation to cover multiple scopes. TCAT will be illustrated using an example on soft capabilities.
\end{abstract}

Keywords: Capability thinking, system thinking, thinking capability analysis technique 


\section{INTRODUCTION}

As the environment we operate within becomes more complex, our organisations respond in a like manner. Missteps and unintentional negative consequences become the order of the day when we no longer understand not only the environment we interact with but also the systems we operate. Thus it becomes increasingly important for us to develop a method of thinking and structuring this thinking that enables us to not only understand ourselves and our interactions but also to ensure that we retain control of our purpose and thus direction. It is this purposeful control in understanding and developing organisations that lead us to propose why organisations need to augment Systems Thinking with CApability Thinking (CAT).

This paper will firstly explore the contemporary understanding of Systems Thinking before introducing the reader to CAT. These two methods will be compared and weighed and finally a conceptually simple methodology (Thinking-Capability Analysis Technique), based on the generationally sturdy Functional Analysis System Technique (FAST) will be presented as an appropriate tool for CAT.

\section{SySteM ThinKING}

Senge (1997) sees Systems Thinking (ST) as a discipline for examining wholes. ST offers organisations a method to manage complexity. It forces planners and strategists to focus on stakeholders, processes, interactions and causes of poor outcomes, rather than individual agents, system components or interim results. As a paradigm, it moves away from the belief that the dynamics of the whole can only be understood from the properties of the parts to a more holistic view. This holistic view attempts to think of the world in terms of many systems interacting together. Each system is a group of components interacting as part of a process. A nested view of systems would see each component as a system in its own right; thus, a system-of-systems approach emerges.

For example, the banking system consists of all banks and the transactions that flow among themselves. It may include other organisations such as financial planning organisations, investors, creditors, and so forth. Should the line (system boundary) be drawn for the banking system around banks only to form the banking circle? Should it move to include investors and creditors? Should it move to include government organisations? This is the first advantage in system thinking, what is the system and where should the circle be drawn? This advantage enables us to scope problems and context using the specific boundaries we draw around a group of things we call a system. However, this advantage comes with a cost. It establishes cognitive biases where an analyst focuses on what is inside the circle called a system and considers everything else outside as the environment (the systems that she can't control). The circle becomes the focus.

Defining the focus creates clarity in the analysis. There is little doubt as to the boundaries and any investigation is thus inherently limited. However where is the utility of the system? A system only becomes useful when it has a purpose. We know the process and the elements within the system but what is the purpose? The answer would depend on who you ask. Put simply, the purpose of a system is defined in the eyes of the beholder. Change the beholder, and the purpose may change. Another advantage of system thinking is that it gives the analyst means to distil the purpose of a system from stakeholders.

Again, the advantage comes with a cost. Control has been lost as the purpose of the system is now defined by an external observer. The bias that comes from the observer is defined by the invested interest the observer has in the system. Change the observer, and we may get a different purpose that we did not even think of. The implication of that is we may fall into a negative risk trap, wherein an external observer sees the purpose in such a way that negatively impacts our objectives. An external observer who wishes to profit from the system would see the banking system as an ideal system to move money around and create a fraud network. Today we know this, but at some point in history, designers of the banking system did not necessarily think of this possibility because they could not necessarily predict all possible purposes that external entities will see about the system; thus, their risk assessment would fail to capture these unknown purposes.

Ulrich Ulrich (2012) states that “...each school of systems thinking tends to give an overly narrow picture of the others, as if the reality of problem situations could ever be captured through a merely quantitative perspective (a caricature of hard system thinking) or a merely subjective perspective (a caricature of soft systems thinking)." Our position is therefore not criticizing system thinking as a field, but offer a critical assessment of many of the methods used by system thinkers. 


\section{Capability Thinking}

CAT offers a remarkably different perspective. The first author established this concept within a course he designed: Capability Options Analysis. Over eight years of teaching the course saw the concept evolve to become a complete framework that expands and improves on System Thinking. In its complete form, CAT starts from strategic vision down to resources. However, for the sake of comparison with ST, we will focus the discussion on a limited version of CAT.

CAT asks eight critical questions to create an understanding of the system and its place within the environment. The first two questions need to be answered simultaneously (1) what are the set of outcomes that we want to achieve in which time-frame and in which contexts? and (2) what are the values of these outcomes to the organisation? These outcomes are also known as effects. For example, the outcome might be that we would like to make the national economy prosper over a specific timeframe as this would in turn lead to an increased national robustness to withstand external shock events.

The third question that CAT asks is what are the set of functions ${ }^{1}$ we would like to work with to achieve this effect? This sort of functional analysis provides a basis to take some inputs and transform them into some specific outputs. We may choose two functions for the example above: an investment function and a job creation function. Through proper functional analysis, each function can be divided into sub functions. The investment function may get divided into an inflation management function (to control inflation and provide the authoritative power over actors who may abuse the situation), a lending function (to inject money into the society), and a financial planning function (to attract money to the financial sector). The job creation function may get divided into a lending function (to provide financial support to organisations to create jobs), an innovation function (to create novel opportunities that require employees), and a training function (to develop new skills in the jobs force). Intentionally, we have used the example above to demonstrate that sub-functions of different functions can overlap; thus, a sub-function can serve multiple functions.

The fourth question that CAT asks is what are the options we can use to build the capacity to deliver these functions so that we are able to deliver the effect? This is what we will call a capability. The simple definition for a capability is the capacity to deliver an effect. We can expand this definition based on the discussion above by defining a capability as the capacity to deliver, perform, and sustain functions to deliver intended effects. This fourth question is tightly coupled with the fifth question: what are the building blocks that need to be in place to build and sustain this capacity? These are known as the Fundamental Inputs to Capabilities (FICs). Each FIC provides a unique capability in its own right; thus, the definition of a capability is a nested one in a similar way to the definition of a system.

While defence has its own FICs to suit its unique operational design, we put forward that FICs are merely a convenient labelling of specific resource combinations and are thus only contextually useful. To this end we have designed the following eight FICs to be generic enough to be suitable for any capability - be it civilian or military, hard or soft. Each FIC is divided further into sub-FICs.

- Personnel: Raise-Train-Sustain. This FIC covers the cycle of recruiting and maintaining people. Training covers all type of training (individual and group/joint) required for skilling and improving competency of people.

- Platforms: Machines-Models-Processes. Platforms are used here in general terms. They cover tools, machines, as well as soft platforms such as models and processes required for sustaining the functioning of a soft systems (soft power).

- Organisation: Structures-Responsibilities-Accountabilities-Contestability. This FIC covers the internal support and architecture behind the bureaucratic system and governance of the whole as a system.

- External support: Physical-Information-Cognitive-Social-Financial. This FIC covers the external dependencies that impact the operation of the capability from physical infrastructure, information infrastructure, perceptions, the social system where the capability is sitting, and the financial system within which the capability operates.

- Knowledge: Acquire-Innovate-Compile-Create. This FIC covers the know-how required to operate and sustain the capability, ranging from how to acquire existing knowledge, innovate, compile and create

${ }^{1} \mathrm{~A}$ function is a generic statement of what needs to be accomplished without specifying the means hence it can be used to describe all things, products, processes, services and procedures. 
new knowledge.

- Cyber: Infrastructure-Information-Spectrums. With the increase reliance on technologies within organisations, Cyber, or the electromagnetic spectrum within which the organisation operates become a FIC to almost every hard and soft capability within an organisation. This FIC covers the physical infrastructure required for the Cyber domain, information as an asset, and spectrum management.

- Facilities: Land, Buildings, Maintenance-Working-Training. This FIC represents the physical infrastructure (lands and buildings) holding and/or hosting the organisation as well as the maintenance of these assets, the facilities for creating a working environment and facilities for training.

- Supply Chains: Logistics-Internal-External. This FIC covers logistics - the blood vessels of any organisation, as well as internal and external flow of materials and information.

The sixth is: what are the resources required to deliver the capabilities defined above? The classic resources (People-Capital-Land-Knowledge) are used to spell out what is needed to deliver the capabilities (Abbass, 2014).

The seventh question is related to the cost: What is the total cost of the capability? The total cost is not just the cost of the resources, but also include the cost for putting the resources together to deliver each FIC, and putting the FICs together (through processes, etc) to deliver the capability. It is important to note here that sustainment cost is already included since the first question we asked defined the need for a capability to deliver an effect within some specific contexts and for a specific timeframe.

The eighth and last question that we need to ask is: does this represent value-for-money? In other words, we need to be clear on the benefit-to-cost analysis.

Benefits are defined through the second question above (value of desired effects to the organisation), while costs are defined in the sixth question above (resources). Depending on the answers to the last two questions, we may need to return and revisit one or all of the questions above.

\section{How doES CAT COMPLEMENT EXISTING ST METHODS?}

First, a capability definition departs from a system definition in that the purpose of a capability (effect generation) is an integral part of the definition; that is, a capability can't be defined in isolation of the effects it is meant to generate. A capability is the capacity to generate an effect. The type of desired effects a capability is intended to generate needs to be part of the design and should not be merely left for an external observer's judgement. Control now rests with the organisation as they assume responsibility for the effect rather than an observer as is the case for Systems Thinking.

Second, the boundaries for a capability are defined by measurable and intended effects, not by a set of artificially imposed boundaries as in the case of a system definition, where boundaries are defined by the bias an analyst has on what falls within and outside a system. This makes the capability definition so flexible. The organisation can design many different ways to deliver the effect. Systems can still be defined however the organisation is also able to design what is influential within the previous environment.

Third, CAT is based on proper functional analysis that maps the inputs to outputs. When assessing risks, we are not stuck within a particular view of the world or a particular cognitive bias. We still have a bias based on our know-how in conducting the functional analysis properly, but as we learn to overcome this bias, we focus on the capacity embedded within a capability rather than the sometimes subjective view of what a system purpose is or is not.

Fourth, from an organisation perspective, CAT starts with the objectives of the organisation. It forces the organisation to clearly think about the reason of its existence, the effects it needs to generate, and how these effects support its mission and vision. CAT is therefore more objective and leaves out most of the subjectivity that is inherent in ST.

Fifth, CAT is more suitable for grass-root design and for reverse-engineering systems, while classical ST is more suitable for diagnosis and retrofitting analysis. While ST brings the stakeholders inside the analysis, CAT brings the stakeholders inside the system boundary. 


\section{Function Analysis System TechniQue}

Function Analysis System Technique (FAST) was developed by Charles W. Bytheway in 1964(See Bytheway $(1972,2007))$. It is a decision aiding technique mostly used by system engineers to map out the functional interdependency in a system. The main advantages of FAST includes: it is a visual representation that makes it easy for an analyst to communicate the analysis; it captures the how and why together; it can be interrogated quickly to discover gaps in logic; and it allows for team work to evolve the analysis.

Shillito and De Marle (1992) describe FAST as employing five basic steps. (1) Use verb-noun function descriptions to define the functions performed by the product and its components. (2) Create a logical relationship using three simple questions: Why are we doing this? How are we achieving this? And when we do this, we also do what? The use of these questions allows for the building of the model. Figure 1 illustrates. (3) "Logic structure is verified in the reverse direction." (4) a critical function path is identified, and (5) the limits are defined. One of the greatest advantages of FAST was its ability to provoke discussion and generate ideas.

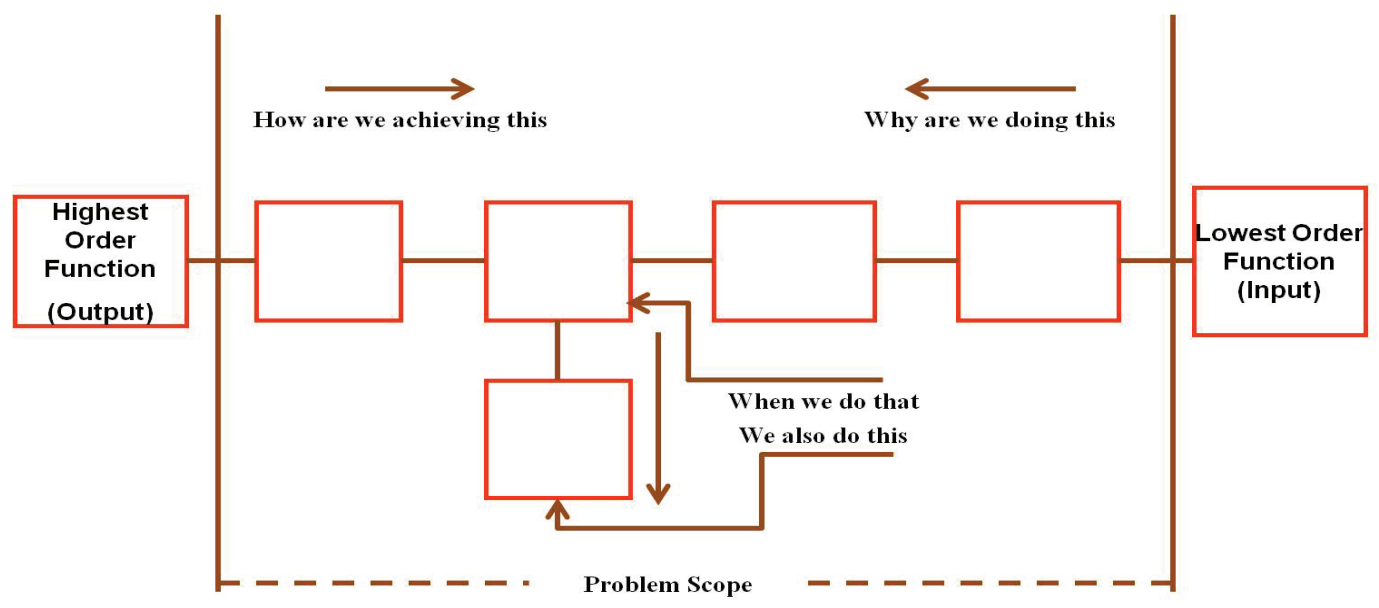

Figure 1. A Conceptual Diagram of the Function Analysis System Technique.

\section{Thinking Capability Analysis Technique (TCAT)}

Thinking Capability Analysis Technique (TCAT) (See Figure 2) is a modification of the FAST that is specifically designed to understand the capability development required to achieve an effect. It is this holistic approach focusing on enablers that differentiates TCAT from FAST. As previously discussed FAST is designed to elicit understanding of a system which is, by definition, constrained by the analyst. TCAT is an adaption of a hierarchical exploration methodology that it allows the analyst to understand the requirements in a capability development context.

The lexicon is important as it provides focus for the analyst to understand what is required to enable or generate the effect. FAST is limited to looking at how functions are specifically achieved within the system while TCAT extends this concept to look at what enablers are required to achieve the effect. Thus the analyst is asked what capability enables the resulting effect. The use of Active Verbs + Measurable Nouns remain as an integral part of this lexicon though the key change in the lexicon is the use of the term enable.

Visually, TCAT is similar to FAST. There is a dual flow from left to right and right to left. The rectangles (denoting concepts, effects, functions, capabilities, FICs, or resources) on the right enable those to the left. The vertical lines denote either ownership or concurrency of activity. A vertical dashed-line is used to articulate who is responsible for the enabler. For example in Figure 3, Social Welfare is responsible for Protecting the Society whilst Defence is responsible for Protecting Countries. A vertical solid-line on the other hand indicates a concurrent enabler. Here the interpretation is "when". For example when we Synthesize Capabilities we also Build Capabilities.

Figure 4 also illustrates an additional advantage TCAT has over FAST and that is it is able to combine multiple scopes. As already mentioned effects are generated by capabilities and it is usual for capabilities to combine 


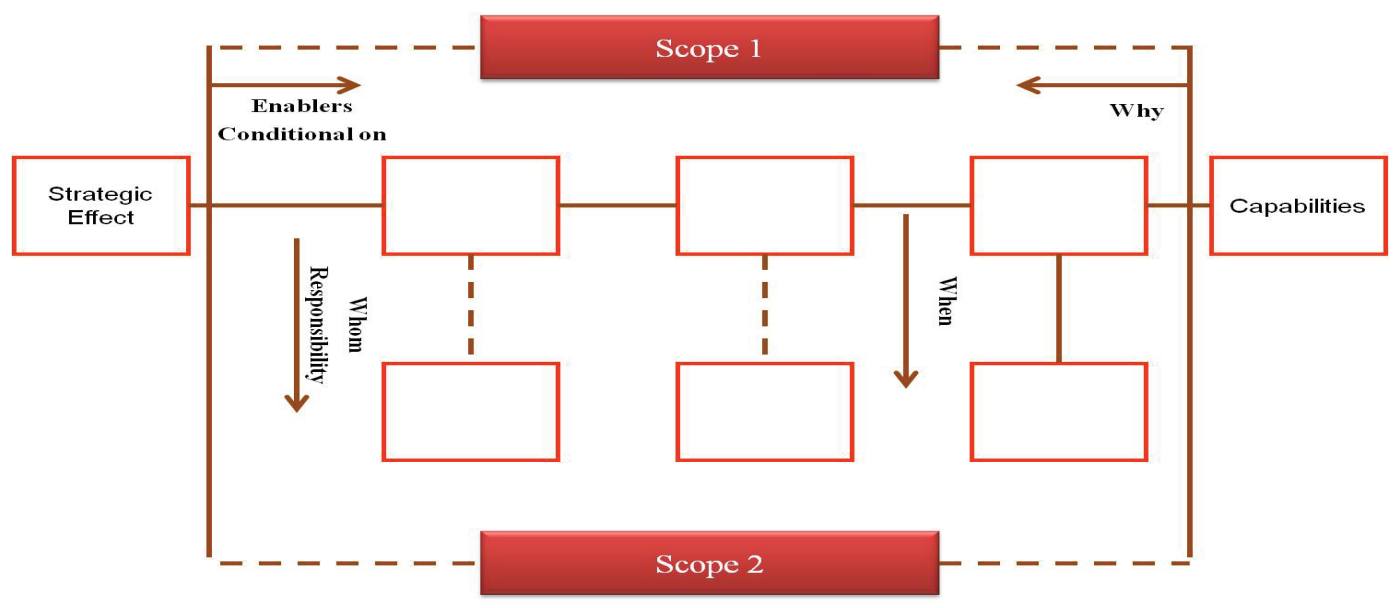

Figure 2. A Conceptual Diagram of the Thinking Capability Analysis Technique.

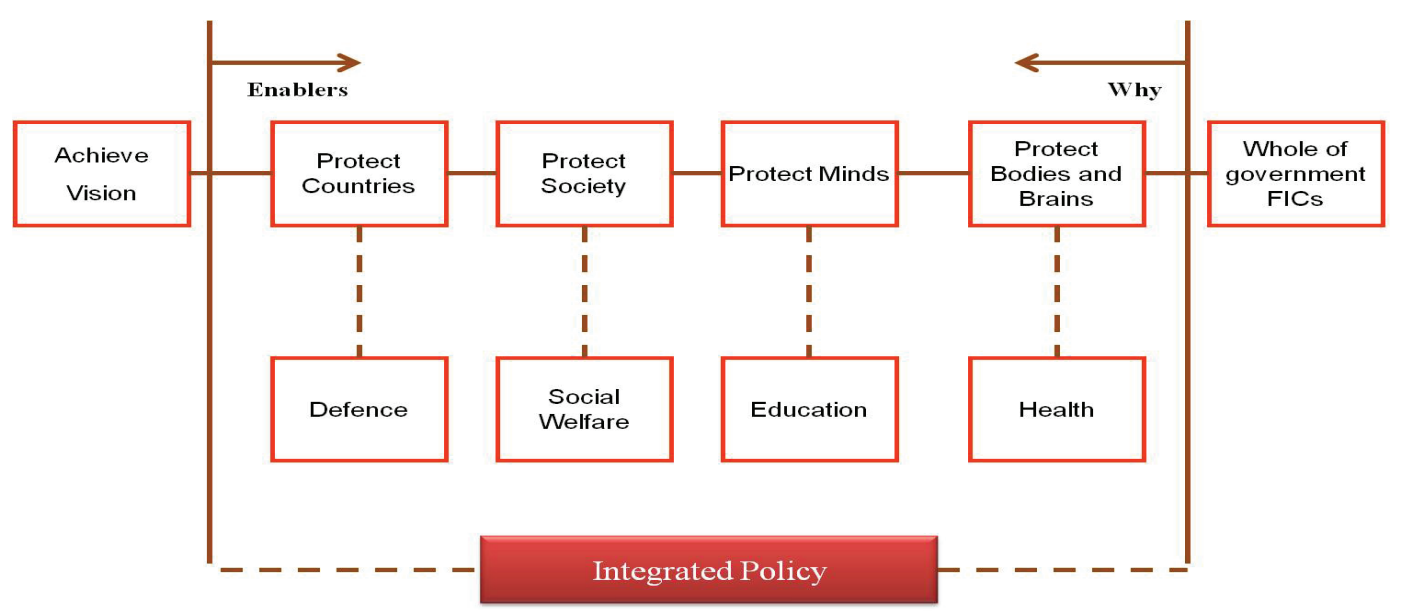

Figure 3. Example of Thinking Capability Analysis Technique for National Security.

(as they are nested) to form meta-capabilities. In this case the two meta-capabilities are described as Hard Capabilities and Soft Capabilities. However the split does not always have to be on the type of capability. Unlike FAST, the TCAT analysis is not limited to a single system or entity.

The actual employment of TCAT is deliberately flexible to allow it to maximise on the positive characteristics of FAST. For instance the actual design of a TCAT for a specific effect allows the analyst to question the required enablers and it is this inquisitive process that provides the most benefit. A benefit that would disappear if the process became rigid and formulaic. Normally the TCAT would be used in a similar manner to backcasting. The required effect is generally provided through organisational vision or mission and the base level resources available are also generally known. From there it becomes a simple matter of linking resources to effects through a series of enablers.

The development of the example in Figure 3 is based on a holistic vision of security. The resources available to the government, in this case, are grouped into combinations and conveniently labelled as FICs. This simple accounting process generally makes it easier for organisations, in this case the National Government, to understand the building blocks they have to play with (policy tools). The building blocks are used to enable specific tiered capabilities and effects. For instance Protect Bodies and Brains (physical) is required to enable Protect Minds. The reason we Protect Minds is to Protect Society. In this case, there are no concurrent capabilities though one could extrapolate at least two other soft capability lines and effect lines based on (1) environment and (2) regional security or trust. The dashed-lines indicate the responsible departments within the organisation for ensuring the capability is achieved. 


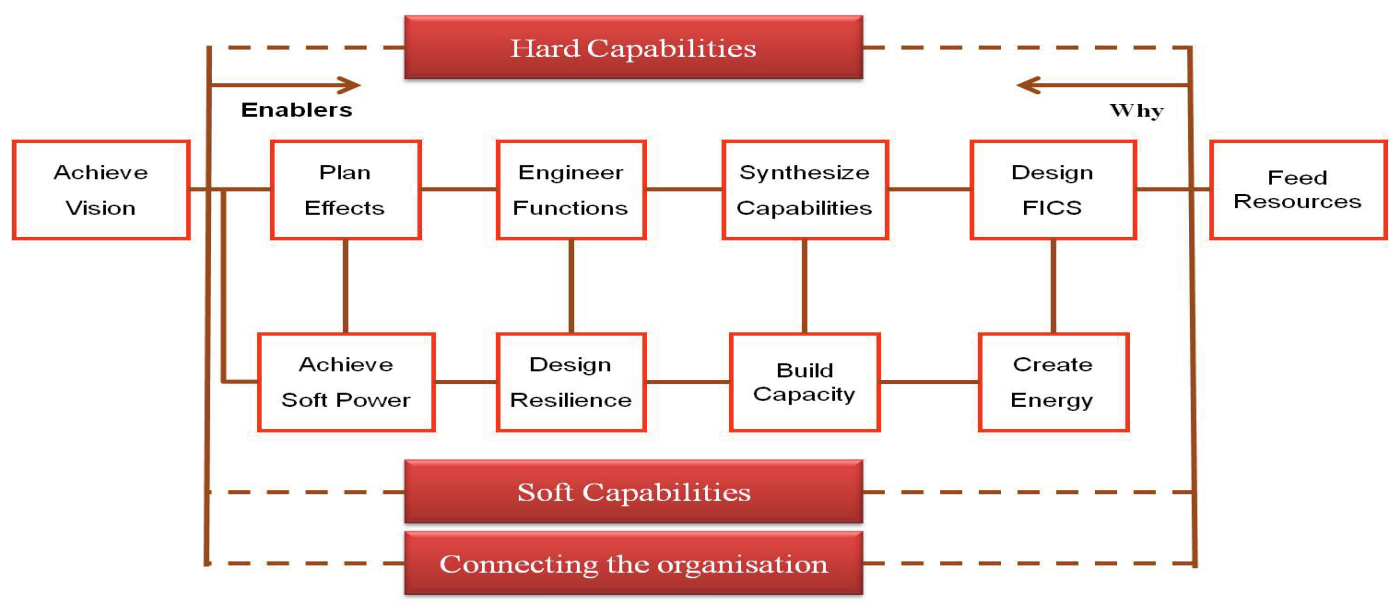

Figure 4. Example of Thinking Capability Analysis Technique for A Capability-Based Strategy Framework.

The development of this TCAT allows the organisation to understand the full resource requirement and capability development bill. This in turns allows an understanding of the inherent resource conflicts that will occur should each department try to maximize their allocated capability. Simulation and modelling would then be required to elicit cost-benefit ratios and value for money (the final questions used in CAT). Another example of TCAT demonstrating a capability-based strategy framework is shown in Figure 4.

\section{Conclusion}

This paper sought to highlight the costs in using a Systems Thinking approach and proposed a complementary perspective - CAT. CAT allows (1) the purpose of the capability to be integral to its definition and design; (2) the system boundaries are defined by the intended effect not through an inherently biased judgement; (3) it is based on proper functional analysis that maps inputs to outputs; and (4) by starting with the objectives of the organisation, it forces the organisation to clearly think about the reason of its existence, the effects it needs to generate, and how these effects support its mission and vision.

Finally we presented a conceptually simple method, based on FAST, that allows for comprehensive CAT in the analysis of a system. Thinking-Capability Analysis Technique (TCAT) allows the organisation to understand the full resource requirement and capability development bill. While visually similar to FAST, TCAT allows for multiple scopes and is focused on capability and effects rather than simple functions without an overarching intent.

\section{REFERENCES}

Abbass, H. A. (2014). Computational Red Teaming: Risk Analytics of Big-Data-to-Decisions Intelligent Systems. Springer.

Bytheway, C. (1972). Function analysis system technique. In Society of American Value Engineering, 1965 International Conference Proceedings.

Bytheway, C. W. (2007). Function Analysis Systems Technique Creativity and Innovation. J. Ross Publishing.

Senge, P. M. (1997). The fifth discipline. Measuring Business Excellence 1(3), 46-51.

Shillito, M. L. and D. J. De Marle (1992). Value: its measurement, design, and management. John Wiley \& Sons.

Ulrich, W. (2012). Operational research and critical systems thinkingan integrated perspectivepart 1: Or as applied systems thinking. Journal of the Operational Research Society 63(9), 1228-1247. 